\title{
Soft Pushing Operation with Dual Compliance Controllers Based on Estimated Torque and Visual Force
}

\author{
Abdul Muis Student Member (Keio University) \\ Kouhei Ohnishi Senior Member (Keio University)
}

Keywords: pushing operation, compliance controller, mobile manipulator, reaction torque observer, robot vision, artificial marker

Future robot is expected to accomplish more and more complex task, especially to work with human life. Indeed, the robot should be compliance with environment either human being. An interesting application in such an issue is pushing operation.

Generally, the pushing operation consists of "approaching, touching, and pushing". However, most researches in this field are dealing with how the pushed object follows the object's trajectory. Obviously, one needs to considers the damage that may happened to the object during "touching" stage, especially if pushing operation is done by robot with high momentum.

Indeed, this paper proposes a novel structure for soft pushing operation with dual compliance controllers. The robot motion will be controlled in three stages of pushing operation; approaching, touching and pushing. Although a compliance control provides robot with affinity and adaptability to work in open environment, a compliance control while touching is not enough for robot with high momentum. In which, the robot should slow down while approaching the object. Fig. 1 shows the pushing operation without any compliance behavior. Although the position response tightly followed the position command, the robot does not care whether it hit any damage on the object. The robot treats the object as an obstacle to be vanished as long it follows the position command.

The compliance controller in this paper is not only for touching stage, but also for the whole stages; approaching, touching and pushing. Generally, compliance control is a control system with trajectory compensation so that an external force may be followed. The first compliance controller in this paper is driven by estimated external force based on reaction torque observer. This controller is

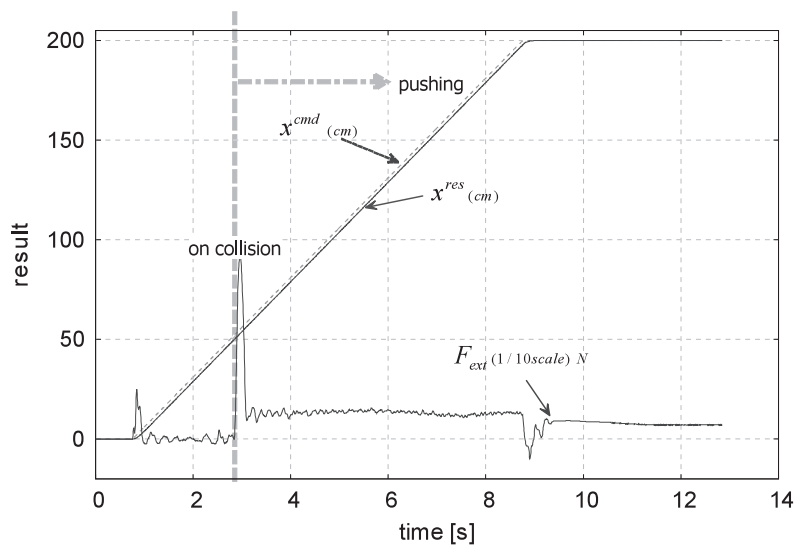

Fig. 1. Pushing operation without any compliance behavior projected to compensate contact sensation. The second controller compensates non-contact sensation. Surely, a force sensation from force sensor either reaction torque observer is available just after the robot touched the object. It is too risky to damage the object while touching. Indeed, a visual sensor is considered as non-contact sensation. Instead of using visual information as command reference, the visual information such as depth in this paper, is treated as visual force for the second compliance controller.

A heavy mobile manipulator and heavy object are considered in this paper. A chopstick is attached on the object side to show the effectiveness of the proposed method. In overall, both compliance controllers adjust the mobile manipulator command reference to provide soft pushing operation.

Based on experimental results, dual compliance controllers is not enough. Although the soft pushing operation is realized, the position response could not follow the position command. Indeed, a modification on compliance controller reference is applied. The objective of this modification is to make sure the robot still follow the position command while keeping soft touching and pushing. Obviously, the soft pushing is conducted by giving compensation on that time. Once the robot slow down after touching the object, a compliance behavior can be released gradually. This is realized by multiplication of compliance controller reference with the robot speed.

The soft pushing operation response based on the proposed method is shown in Fig. 2. The position response is move slowly compared to the position command. Finally, in the end the compliance behavior is released. The X-cmp-env and X-cmp-vis responses become zero in the end. Finally, the reponse position remain steady as commanded.

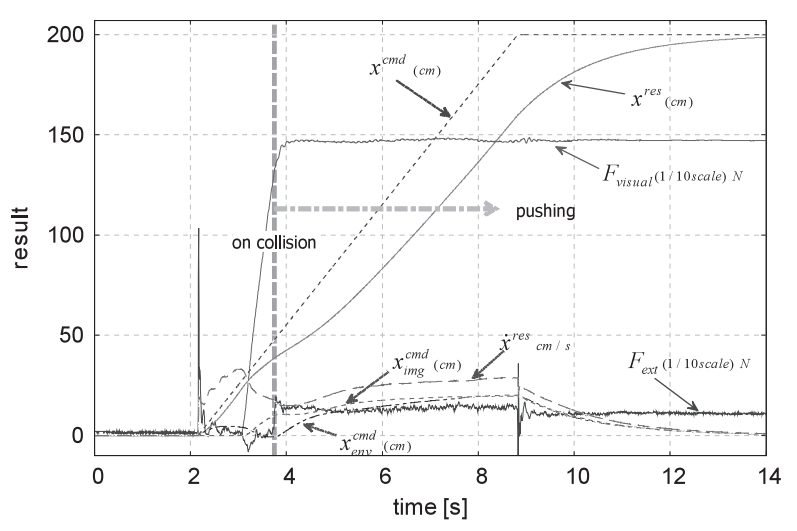

Fig. 2. Pushing operation with dual compliance controllers 


\title{
Soft Pushing Operation with Dual Compliance Controllers Based on Estimated Torque and Visual Force
}

\author{
Abdul Muis* Student Member \\ Kouhei Ohnishi* Senior Member
}

\begin{abstract}
Sensor fusion extends robot ability to perform more complex tasks. An interesting application in such an issue is pushing operation, in which through multi-sensor, the robot moves an object by pushing it. Generally, a pushing operation consists of "approaching, touching, and pushing" (1). However, most researches in this field are dealing with how the pushed object follows the predefined trajectory. In which, the implication as the robot body or the tool-tip hits an object is neglected. Obviously on collision, the robot momentum may crash sensor, robot's surface or even the object. For that reason, this paper proposes a soft pushing operation with dual compliance controllers. Mainly, a compliance control is a control system with trajectory compensation so that the external force may be followed. In this paper, the first compliance controller is driven by estimated external force based on reaction torque observer ${ }^{(2)}$, which compensates contact sensation. The other one compensates non-contact sensation. Obviously, a contact sensation, acquired from force sensor either reaction torque observer of an object, is measurable once the robot touched the object. Therefore, a non-contact sensation is introduced before touching an object, which is realized with visual sensor in this paper. Here, instead of using visual information as command reference, the visual information such as depth, is treated as virtual force for the second compliance controller. Thus, having contact and non-contact sensation, the robot will be compliant with wider sensation. This paper considers a heavy mobile manipulator and a heavy object, which have significant momentum on touching stage. A chopstick is attached on the object side to show the effectiveness of the proposed method. Here, both compliance controllers adjust the mobile manipulator command reference to provide soft pushing operation. Finally, the experimental result shows the validity of the proposed method.
\end{abstract}

Keywords: pushing operation, compliance controller, mobile manipulator, reaction torque observer, robot vision, artificial marker

\section{Introduction}

Among common human activities that are being adopted in robot application is pushing an object. Pushing operation is just a simple trajectory planning for pure position control with prior knowledge on object displacement from the robot. Mainly pushing operation is conducted in three steps; "approaching, touching, and pushing" (1). Nevertheless, although more pushing power can be attained by the robot, this operation is still limited to certain degree compared to human being. In which, human is able to sense the object and its surrounding perfectly so that human may apply certain actions to make the object moved as desired.

Recently, a robot is expected to realize more and more complex tasks, the need to take the advantage of multiple sensors in robotics system becomes increasingly important. In addition, the future robot is required to work at human environment, i.e., the robot should be compliant with environment and human being. Pushing operation is an interesting application in such an issue. In which, multi sensor is often used to assure the object being pushed as desired $^{(1)(3)-(6)}$. Verma ${ }^{(3)}$ performs box-pushing by a simple mobile robot with environment-embedded sensors. The robot pushes a detected object based on pose information obtained

\footnotetext{
* Keio University Dept. of System Design Eng.

3-14-1, Hiyoshi, Kohoku-ku, Yokohama 223-8522
}

from sensors. Here, the accuracy of the push operation is correlated to the number of used sensors to determine the object pose. In contrast, $\mathrm{Yu}^{(5)}$ estimate object inertia based on two force sensors on robot tool-tip to decide which object side should be pushed. In similar way, Kawasa ${ }^{(6)}$ estimate object inertia with PSD sensor. Based on the object inertia, the pose of redundant manipulator is controlled, so that the object moved as desired trajectory.

Multi sensor is also considered in this paper. However, this paper not only ensures the object being pushed as desired, but also considers the implication to robot/object surface at touching stage. Mainly, the robot surface/tool-tip hits an object at touching stage. For some cases, the robot may deal with sensitive object, or sensitive device mounted on robot tool-tip such as force sensor. Obviously, certain actions should be done to avoid damage on these stuffs, especially on collision. One may set the robot with slow motion while approaching until touching the object. In which, reduces pushing performance. In different way, with prior knowledge on precise object displacement from the robot, one may set the robot to run and stop close to the object. However, it is hard to obtained precise displacement on real environment. For that purpose, this paper proposes soft pushing operation while keeping pushing performance.

Approaching, touching, and pushing stages are considered by Katsura et al. ${ }^{(1)}$ during their pushing operation. A compliance controller provides robot with affinity and adaptability 
to work in open environment, utilization during touching is not enough to relax the impact force especially for robot approaching with significant momentum. Obviously, the robot should slow down before touching the object. Therefore, a compliance control is not only required in touching stage, but also required in approaching stage. Practically, if the robot motion is driven by human directly, a soft pushing operation can be realized. Ise et al. ${ }^{(7)}$ develop a bilateral control on two mobile manipulator. The slave robot push the object with respect to motion of master robot which driven directly by the operator.

The soft pushing operation in this paper is realized with dual compliance controllers. These controllers are not only for touching stage, but also for the whole stages; approaching, touching and pushing. Generally, a compliance control is a control system with trajectory compensation so that an external force may be followed. This structure is inspired by Katsura ${ }^{(8)}$. A dual compliance controllers is used to compensate environmental disturbance and human command respectively. In this paper, the first compliance controller is driven by estimated external force based on reaction torque observer ${ }^{(2)}$. This controller is applied to compensate contact sensation. The second controller compensates non-contact sensation. Surely, a force sensation from force sensor either reaction torque observer is available just after the robot touched the object. It is too risky to break the object while touching. Indeed, a visual sensor is considered as non-contact sensation. Instead of using visual information as command reference, the visual information such as depth in this paper, is treated as virtual force for the second compliance controller. In fact, sensor fusion of force and visual sensor has been conducted in most pushing operations $^{(1)(3)(4)(6)}$. Unfortunately, the visual information is used just for robot/object tracking.

Having sensor fusion, the robot will be compliant with wider sensation. The visual information in this paper is provided by high-speed vision sensor running on $250 \mathrm{~Hz}$. The target object is attached with hexagon pattern of artificial markers to reduce the required image processing time. The high-speed hardware performance is provided with simple image processing algorithm based on hexagon pattern of artificial markers that is done in the previous work ${ }^{(9)}$. The vision system is able to obtain the object distance to the vision sensor within $2.5 \mathrm{~ms}$. Here, the distance approximation is utilized as visual force where the distance less than one meter is considered as positive force with opposite direction to robot motion. Thus, whenever the robot approaches the object with certain command reference, the visual force will give a virtual reaction force to the robot. The closer to the object, the more visual force exerted. Basically, this virtual force is similar as potential field. Nevertheless, the compliance controller transforms it moderately through virtual impedance.

In order to show the validity of this paper, a heavy mobile manipulator and a heavy object are considered in this paper. Mainly, a heavy robot moves with significant momentum. Thus, a collision with an object may risk sensitive object. A chopstick is attached on the object side to show the effectiveness of the proposed method. In overall, both compliance controllers adjust the mobile manipulator command reference to provide soft pushing operation.
The rest of this paper is organized as follows; the robot model and the target object are described in Section 2. Later in Section 3, the reaction torque observer is presented. Next, a general view of compliance controller and dual compliance controller in this paper are described in Section 4. A general pushing operation and soft pushing operation are conducted through experiments and discussed in Section 5. Finally, section 6 summarizes this paper.

\section{Modeling}

In this section, the mobile manipulator model and the marked object are described. A mobile manipulator consists of a vehicle part with two independent driving wheels and a multi degree of single link manipulator part as shown in Fig. 1.

2.1 Mobile Manipulator This paper considers the vehicle part of mobile manipulator as the main system, while the manipulator as the supporting system. The vehicle part, known as mobile robot can be modeled as Fig. 2. Let the middle point $P_{0}\left(x_{0}, y_{0}\right)$ of a driving wheel be a mobile robot's control point. The pose of robot is $X=\left[x_{0}, y_{0}, \phi\right]^{T}$. The robot kinematics can be defined by the Jacobian matrix $J_{v}$,

$$
\begin{aligned}
& {\left[\begin{array}{c}
\dot{x}_{0} \\
\dot{y}_{0} \\
\dot{\phi}
\end{array}\right]=\left[\begin{array}{cc}
\frac{R}{2} \cos \phi & \frac{R}{2} \cos \phi \\
\frac{R}{2} \sin \phi & \frac{R}{2} \sin \phi \\
\frac{R}{W} & -\frac{R}{W}
\end{array}\right] \cdot\left[\begin{array}{c}
\dot{\theta}_{r} \\
\dot{\theta}_{l}
\end{array}\right] \ldots \ldots \ldots \ldots(1)} \\
& \dot{x}_{v}=J_{v}(\phi) \cdot \dot{\theta}_{v} \ldots \ldots \ldots \ldots \ldots \ldots \ldots \ldots \ldots \ldots \ldots \ldots \ldots \ldots \ldots \ldots \ldots \ldots \ldots \ldots \ldots \ldots
\end{aligned}
$$

Where,

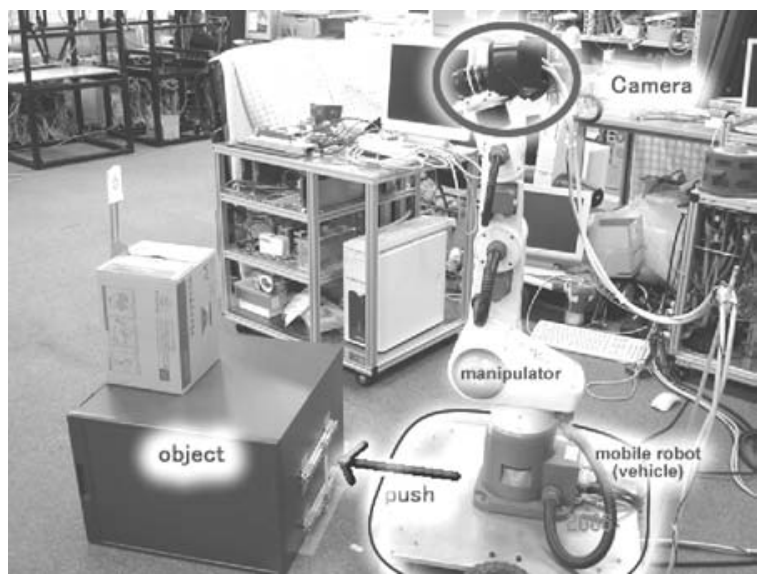

Fig. 1. Mobile manipulator model

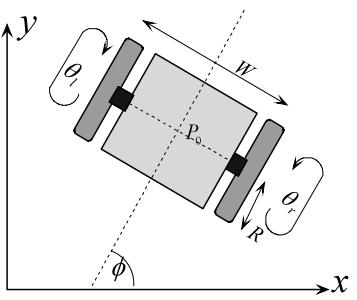

Fig. 2. Model of mobile robot 
$x_{0}:$ The point on $\mathrm{X}$-axes in the world coordinate system

$y_{0}:$ The point on Y-axes in the world coordinate system

$\phi \quad:$ The robot's direction angle

$R \quad$ : The radius of a driving wheels

$W$ : The robot's tread

$\theta_{r} \quad$ : The rotation angle of the right wheel

$\theta_{l} \quad$ : The rotation angle of the left wheel

And the acceleration relation based on derivation of (2), yields,

$$
\ddot{x}_{v}=J_{v}(\phi) \cdot \ddot{\theta}_{v}+\dot{J}_{v}(\phi) \cdot \dot{\theta}_{v}
$$

The second term on the right side is negligible since it has insignificant result compared to the first term. Hence, the equation (3) can be rewrite as,

$$
\ddot{x}_{v}=J_{v}(\phi) \cdot \ddot{\theta}_{v}
$$

Whereas the pseudo-inverse,

$$
J_{v}^{+}=\left(J_{v}^{T} \cdot J_{v}\right)^{-1} J_{v}^{T}
$$

Give the inverse transformation,

$$
\left[\begin{array}{c}
\ddot{\theta}_{r} \\
\ddot{\theta}_{l}
\end{array}\right]=\frac{1}{R} \cdot\left[\begin{array}{ccc}
\cos \phi & \sin \phi & \frac{W}{2} \\
\cos \phi & \sin \phi & \frac{-W}{2}
\end{array}\right] \cdot\left[\begin{array}{c}
\ddot{x}_{0} \\
\ddot{y}_{0} \\
\ddot{\phi}_{0}
\end{array}\right] \cdots \cdots(6)
$$

Here, the joint angle acceleration command for vehicle $\ddot{\theta}_{v}^{c m d}$ can be obtained from the acceleration command $\ddot{x}_{v}^{c m d}$ in the world coordinate system.

2.2 Vision System As described in the preceding section, the system has dual compliance controllers. The first one with estimated torque is described in the following section. The second one with visual force is described in this section. The visual force is a function of estimated distance from the marked object to the camera frame. The camera is attached in the tool-tip of the manipulator driven with two last joints of the manipulator. The rigid connection between the camera and these joints is shown in Fig. 3. These joints and the vehicle part are driven independently. A visual tracking control is applied in these joints to keep the object always in camera view. The vision system not only provides pixel errors of the vertical and horizontal axes on image plane for tracking, but also the approximated distance from the marked object for later used as visual force. In order to shorten the processing time, the marked object is designed as hexagon pattern of artificial marker as shown in Fig. 4. Here, the camera is running within $250 \mathrm{fps}$ or $4 \mathrm{~ms}$ sampling period, while the required processing time of each image is about $2.5 \mathrm{~ms}$.

Having the perspective camera model and assumed the distance between the camera and the marked object is about $1 \mathrm{~m}$, the Image Jacobian based on Scaled orthographic projection can be stated as,

$$
\dot{\tilde{x}}=J_{\text {image }} \dot{x}_{\text {cam }}, \quad J_{\text {image }}=s\left[\begin{array}{ll}
1 & 0 \\
0 & 1
\end{array}\right]
$$

where the scaled projection constant $s$, is obtained through simple calibration ${ }^{(9)}$. Surely, this scaled projection contains lot of noise, especially if the distance between the camera and the object is too close. However, one-meter distance is considered far enough compared to focus length about $2.5 \mathrm{~mm}$.

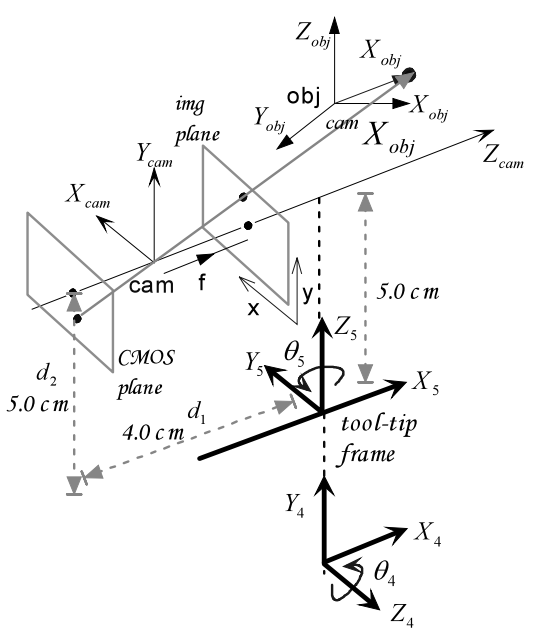

Fig. 3. Camera frame to tool-tip frame model

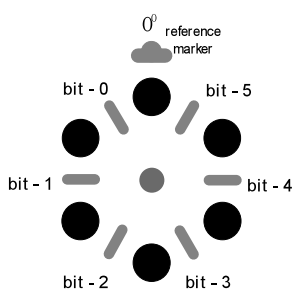

Fig. 4. Hexagon pattern of artificial makers for object model

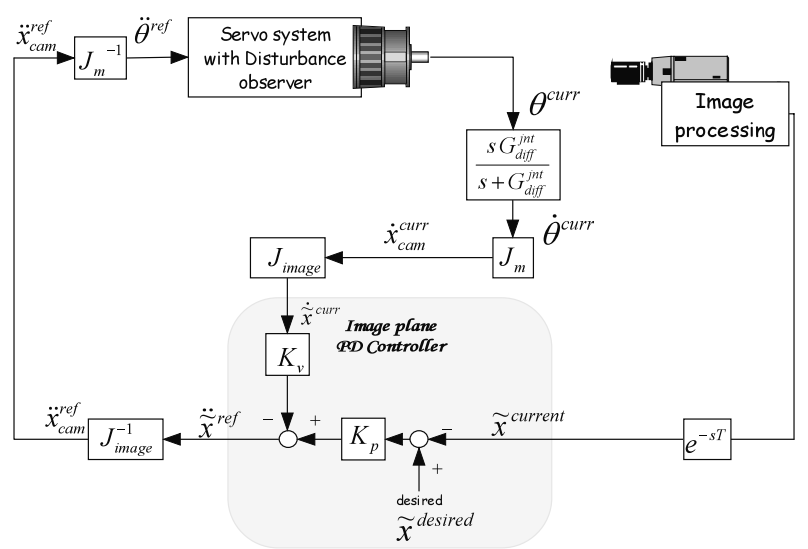

Fig. 5. Visual tracking for the tool-tip control

And the velocity relation between the camera frame to the last two joint (4 and 5) can be expressed as,

$$
\begin{gathered}
{\left[\begin{array}{c}
\dot{x} \\
\dot{y}
\end{array}\right]_{c a m}=J_{m} \cdot\left[\begin{array}{c}
\dot{\theta}_{4} \\
\dot{\theta}_{5}
\end{array}\right] \ldots \ldots \ldots \ldots \ldots \ldots} \\
J_{m}=\left[\begin{array}{cc}
\left(d_{2}+a 4\right) \sin \theta_{5} & -\left(d_{1}-f\right) \\
-\left(d_{1}-f\right) \cos \theta_{5} & 0
\end{array}\right] .
\end{gathered}
$$

The visual tracking control system is developed based on PD control in the image plane. Here, based on (7), yields,

$$
\begin{aligned}
\dot{x}_{\text {cam }} & =J_{\text {image }}^{-1} \dot{\tilde{x}} \\
\ddot{x}_{\text {cam }}^{\text {ref }} & =J_{\text {image }}^{-1}\left(\ddot{\tilde{x}}^{\text {ref }}-\dot{J}_{\text {image }} \dot{\tilde{x}}\right) \\
& \approx J_{\text {image }}^{-1} \ddot{\tilde{x}}^{\text {ref }}
\end{aligned}
$$

The relation between the velocity at camera frame and joint 
velocity is described as (8), and yields,

$$
\ddot{\theta}=J_{m}^{-1}\left(\ddot{x}_{c a m}-\dot{J}_{m} \dot{x}_{c a m}\right) \approx J_{m}^{-1} \ddot{x}_{c a}
$$

Next, the equation on image plane is given as follows,

$$
\ddot{\tilde{x}}^{\text {ref }}=K_{p}\left(\tilde{x}^{\text {desired }}-\tilde{x}^{\text {current }}\right)-K_{v} \dot{\tilde{x}}^{\text {current }}
$$

Thus, the acceleration command on image plane is given as follows,

$$
\ddot{\tilde{x}}^{\text {ref }}=K_{p}\left(\tilde{x}^{\text {desired }}-\tilde{x}^{\text {current }}\right)-K_{v} J_{\text {image }} J_{m} \dot{\theta}^{\text {current }}
$$

From (10), (11), and (13), the acceleration reference for the two last joints is given by (14), the block diagram of this system is shown in Fig. 5.

$$
\begin{aligned}
\ddot{\theta}^{\text {ref }} & =J_{m}^{-1} \ddot{\tilde{x}}^{\text {ref }} \\
& =J_{m}^{-1} J_{\text {image }}^{-1}\left\{K_{p}\left(\tilde{x}^{\text {des }}-\tilde{x}^{\text {curr }}\right)-K_{v} J_{\text {image }} J_{m} \dot{\theta}^{\text {curr }}\right\}
\end{aligned}
$$

\section{Environmental Disturbance Estimation}

In conventional force control, force sensors have been implemented to sense the external force. Mainly, the strain of the strain gauge in force sensor is assumed as external force.

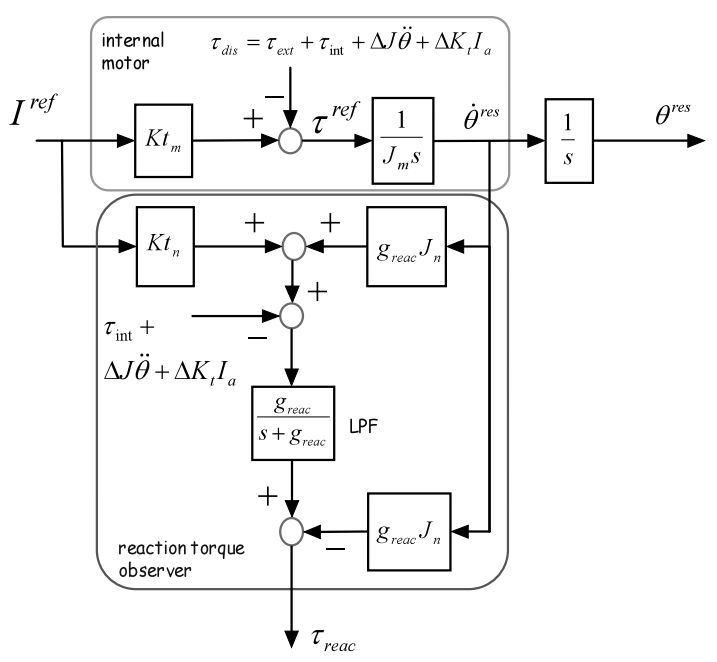

Fig. 6. Reaction force observer

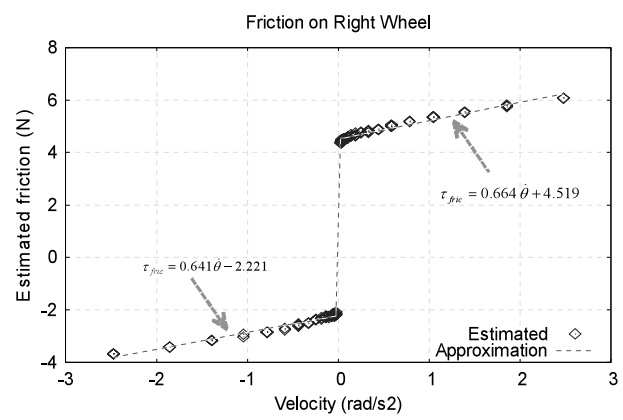

(a) Right Wheel
However, by using reaction torque observer, the external disturbance torque exerted on the motor can be estimated without sensors as illustrated in Fig. $6^{(2)}$.

Estimated reaction torque is as follows;

$$
\begin{aligned}
\tau_{\text {reac }}= & \frac{g_{\text {reac }}}{s+g_{\text {reac }}}\left(I_{a}^{r e f} K t_{n}+g_{\text {reac }} J_{n} \dot{\theta}-\tau_{\text {int }}-\Delta J \ddot{\theta}-\Delta K_{t} I_{a}\right) \\
& -g_{\text {reac }} J_{n} \dot{\theta} \ldots \ldots \ldots \ldots \ldots \ldots \ldots \ldots \ldots \ldots \ldots \ldots \ldots \ldots \ldots \ldots \ldots \ldots
\end{aligned}
$$

where $\tau_{\text {int }}$ is the friction effects including the gravity effects. However for this case, the gravity effect is a steady disturbance, so the gravity compensation has little influence on the contact motion. Assume that the nominal values $J$ and $K t$ are well adjusted, the friction effects can be investigated by some motion tests with constant velocity. In which, the wheels are assigned to move forward and backward at least three times for each constant velocity. The velocity command is decreased on each consecutive loop. The estimated torque of reaction torque observer as described in Fig. 6 but without $\tau_{\text {int }}$, is the estimated friction for current velocity. Later, this estimated friction is used as $\tau_{\text {int }}$ to obtain the external torque. This external torque represents the reaction torque from the environment or the applied force by human or environment to the actuator. Practically, although with the same velocity, the estimated friction may fluctuate due to gear backslash, dissimilar friction on wheel rotation either on floor spot. Nevertheless, the average for each constant velocity can be obtained as shown in Fig. 7 for both wheels. Furthermore, in order to simplify external torque estimation in real-time, the estimated friction can be normalized into viscosity coefficient $D$ and coulomb friction $F_{C}$ as (16).

$$
\tau_{\text {fric }}=D \dot{\theta}+F_{C}
$$

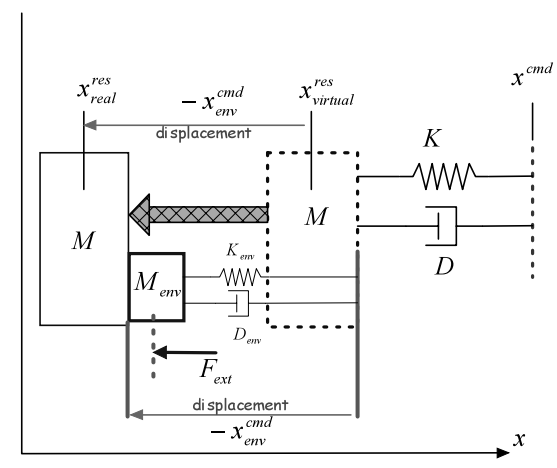

Fig. 8. Compliance control concept

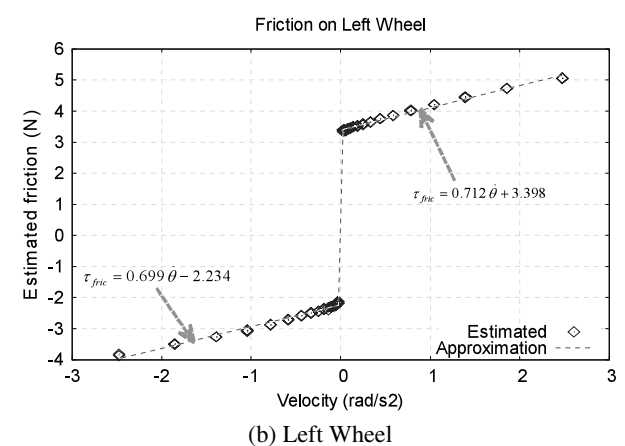

Fig. 7. Estimated friction on wheel (positive value on forward motion) 


\section{Dual Compliance Control}

Compliance control is a control system with trajectory compensation so that the external force may be followed. Stable contact by environment is able to realize through this control scheme, and it become possible to give robot flexibility. The illustration of this concept is shown in Fig. 8.

Let assume there is no external force $F_{\text {ext }}$, the robot may stay in $x_{\text {virtual }}^{\text {res }}$. The mechanical model appears as just PD control. The formulation can be written as,

$$
M \ddot{x}_{\text {virtual }}^{\text {res }}+K\left(x^{r e s}-x^{c m d}\right)+D\left(\dot{x}^{r e s}-\dot{x}^{c m d}\right)=0
$$

Then,

$$
\begin{aligned}
\ddot{x}_{\text {virtual }}^{\text {res }}= & \frac{1}{M}\left\{K\left(x^{\text {cmd }}-x_{\text {virtual }}^{\text {res }}\right)+D\left(\dot{x}^{\text {cmd }}-\dot{x}_{\text {virtual }}^{\text {res }}\right)\right\} \\
= & K_{P}\left(x^{\text {cmd }}-x_{\text {virtual }}^{\text {res }}\right)+K_{V}\left(\dot{x}^{\text {cmd }}-\dot{x}_{\text {virtual }}^{\text {res }}\right) \\
\ldots \ldots \ldots \ldots \ldots \ldots \ldots \ldots(18) & \\
x_{\text {virtual }}^{\text {res }}= & \frac{K_{V} s+K_{P}}{s^{2}+K_{V} s+K_{P}} x^{\text {cmd }} \ldots \ldots \ldots \ldots \ldots \ldots(19)
\end{aligned}
$$

However, due to external force on virtual object $M_{\text {env }}$, the system will have virtual displacement as much as $x_{e n v}^{c m d}$.

$$
x_{\text {real }}^{\text {res }}=x_{\text {virtual }}^{\text {res }}-x_{\text {env }}^{\text {cmd }} .
$$

Then,

$$
x_{\text {real }}^{\text {res }}=\frac{K_{V} s+K_{P}}{s^{2}+K_{V} s+K_{P}} x^{c m d}-x_{\text {env }}^{c m d}
$$

$$
\begin{aligned}
& \left(s^{2}+K_{V} s+K_{P}\right) x_{\text {real }}^{\text {res }}=\left(K_{V} s+K_{P}\right) x^{c m d} \\
& -\left(s^{2}+K_{V} s+K_{P}\right) x_{e n v}^{c m d} \\
& x_{\text {real }}^{\text {res }}=K_{P}\left(x^{c m d}-x_{\text {real }}^{\text {res }}-x_{\text {env }}^{c m d}\right) \\
& +K_{V}\left(\dot{x}^{c m d}-\dot{x}_{\text {real }}^{r e s}-\dot{x}_{\text {env }}^{c m d}\right)-\ddot{x}_{\text {env }}^{c m d}
\end{aligned}
$$

This displacement can be modeled with virtual impedance, as (23). It generates position, speed, and acceleration based on the input force to controller reference. The parameters of this virtual impedance can be changed as well to provide the desired response of force input.

$$
x_{e n v}^{c m d}=\frac{K_{f}}{M_{e n v} s^{2}+D_{e n v} s+K_{e n v}} F_{e x t}
$$

by substituting eq. (23) into (21), yields

Table 1. Compliance control parameters

\begin{tabular}{|c|c|c|}
\cline { 2 - 3 } \multicolumn{1}{c|}{} & $\begin{array}{c}\text { Reaction } \\
\text { Torque }\end{array}$ & $\begin{array}{c}\text { Visual } \\
\text { Force }\end{array}$ \\
\hline$\omega$ & $3.33 \mathrm{rad} / \mathrm{s}^{2}$ & $12.5 \mathrm{rad} / \mathrm{s}^{2}$ \\
\hline$S$ & 0.8 & 0.8 \\
\hline$T_{s}$ & $1.5 \mathrm{~s}$ & $0.5 \mathrm{~s}$ \\
\hline$K_{f}$ & 0.05556 & 1.8744 \\
\hline$M$ & 1.0 & 1.0 \\
\hline$D$ & 5.333 & 20.0 \\
\hline$K$ & 11.11 & 156.2 \\
\hline
\end{tabular}

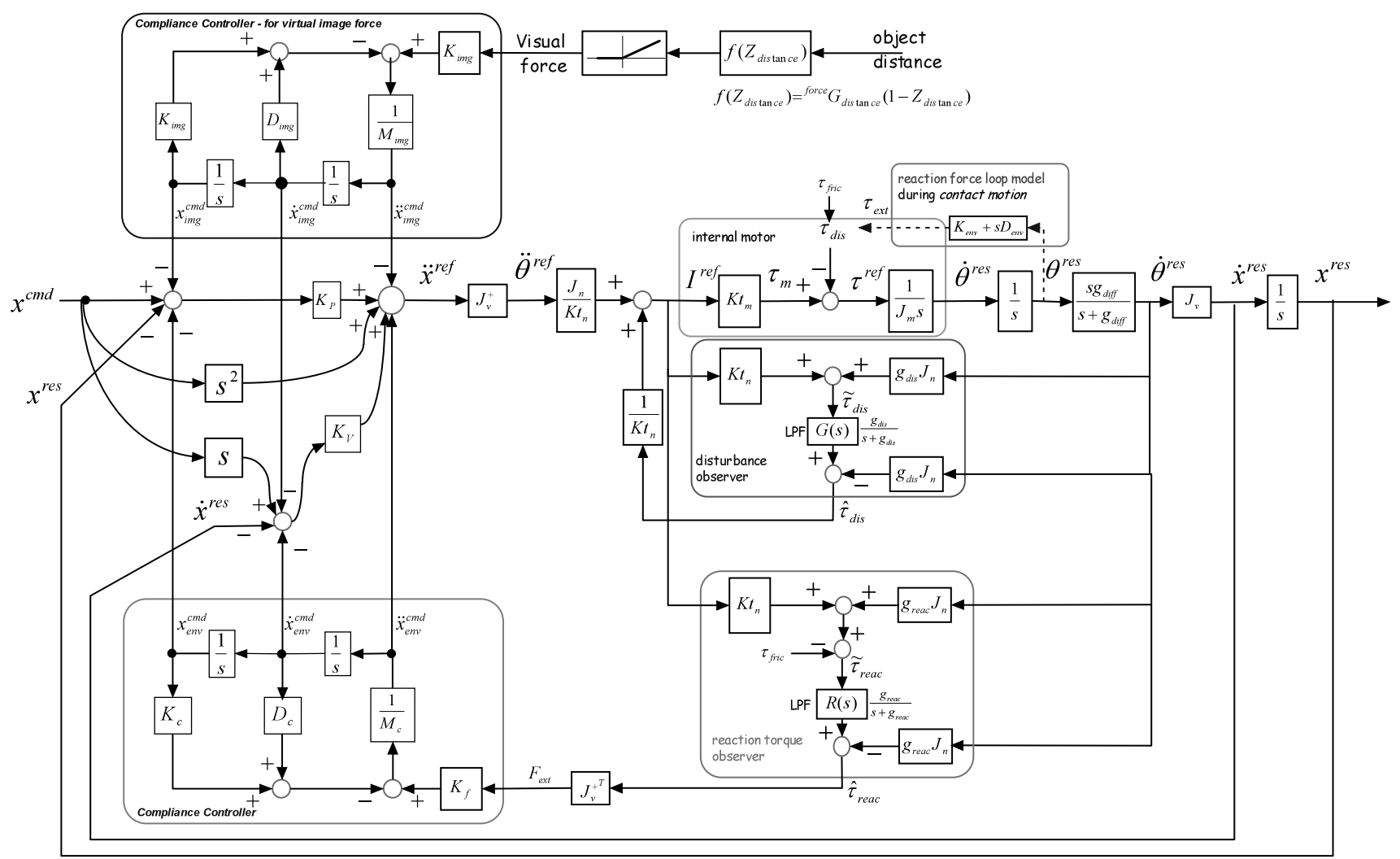

Fig. 9. Dual Compliance control based on estimated torque and visual force for vehicle motion 


$$
\begin{aligned}
x_{\text {real }}^{r e s} & =\frac{K_{v} s+K_{p}}{s^{2}+K_{v} s+K_{p}} x^{c m d}-\frac{1}{M_{e n v} s^{2}+D_{e n v} s+K_{e n v}} K_{f} F_{e x t} \\
& =\frac{K_{v} s+K_{p}}{s^{2}+K_{v} s+K_{p}} x^{c m d}-\frac{\frac{K_{e n v}}{M_{e n v}}}{s^{2}+\frac{D_{e n v}}{M_{e n v}} s+\frac{K_{e n v}}{M_{e n v}}} \frac{K_{f}}{K_{e n v}} K_{e x t} \\
& =\frac{2 \delta_{p} \omega_{p} s+\omega_{p}^{2}}{s^{2}+2 \delta_{p} \omega_{p} s+\omega_{p}^{2}} x^{c m d}-\frac{\omega_{f}^{2}}{s^{2}+2 \delta_{f} \omega_{f} s+\omega_{f}^{2}} C_{f} F_{e x t}
\end{aligned}
$$

where the system response can be changed based on the desired frequency and damping factor as,

$$
\begin{array}{rlrl}
\omega_{p}=\sqrt{K_{p}} & \omega_{f}=\sqrt{\frac{K_{e n v}}{M_{e n v}}} \\
\varsigma_{p}=\frac{K_{v}}{2 \sqrt{K_{p}}} & \varsigma_{f}=\frac{D_{e n v}}{2 \sqrt{M_{e n v} K_{e n v}}} \\
C_{f}=\frac{K_{f}}{K_{e n v}} \ldots \ldots .
\end{array}
$$

Hence, the compliance control extends the robot behavior to be compliant to environment. In other words, the mobile robot will be cooperative, not harming human neither environment within specified virtual impedance.

However, the prescribed compliance above, only works on contact state. Although the robot will be compliant once it hit something, it is too risky to let sensitive object broke on collision. For that reason, this paper employs dual compliance controllers. The first compliance controller is driven by estimated force $F_{\text {ext }}$, which is a multiplicative result of transpose pseudo-inverse Jacobian and estimated torque of both wheels. Later, this controller is applied to compensate contact sensation. This compensation is emerged during touching and pushing stage. On the other hand, the second controller compensates non-contact motion based on visual force. This compensation is emerged during approaching and touching. This visual force is defined based on estimated object distance, which is provided by the visual tracking control on the manipulator part as described in Section 2.2. Differ to most visual feedback in recent robotics applications that is applied as position or speed command reference, this paper utilizes visual information as virtual force. Practically, the visual force is similar as potential field. Nevertheless, the compliance controller transforms it moderately through virtual impedance.

The parameters for compliance control can be designed by parameters as shown in equation (26). In this paper, the desired requirement of compliance controller based on reaction torque observer is defined to have settling time less than $1.5 \mathrm{~s}$, with damping ratio of 0.8 . In addition, $1 \mathrm{~N}$ estimated force is defined to give $0.5 \mathrm{~cm}$ displacement. The adopted impedance parameters for reaction torque observer and visual force are shown in Table 1. Since the visual system is independent from the vehicle part, the response system is defined faster with $0.4 \mathrm{~s}$ settling time. Practically, the damping ratio is defined as close to critically damped system. Whereas the settling time is defined as desired. Sure, it can be set sorter, but due to system constraint such as gear backslash, it may lead to instability.

\section{Experiments}

As described in preceding sections, a pushing operation is conducted in this paper. Here, the robot is assigned to move forward approaching the object. Of course, the robot path is not limited to forward motion. It depends on where the object is located. The robot is represented by mobile manipulator. Two control structures are conducted in this robot. The main system is the vehicle part that is applied with dual compliance controllers. The manipulator part behaves just a supporting system to provide visual information as visual force. The object is represented by a bookshelf with nominal mass about $20 \mathrm{~kg}$. The object displacement is not defined precisely to show the effectiveness of non-contact sensation. Nevertheless, the robot should have enough space to attained speed on approaching stage. In addition, due to visual system constraint in this paper, the object is placed less than $60 \mathrm{~cm}$ from the robot. Mainly, the object displacement is set about 40$50 \mathrm{~cm}$ in this paper. A chopstick is mounted in front of the object to show the effectiveness of the softest level. The initial system condition is illustrated in Fig. 1. Here, the vehicle is assigned to go forward with $30 \mathrm{~cm} / \mathrm{s}$ and push the object. The final position command is $2 \mathrm{~m}$ from the initial position. Practically, the X-slope or the speed for pushing operation is not limited to $30 \mathrm{~cm} / \mathrm{s}$. Unfortunately, due to maximum speed of the vehicle part around $32 \mathrm{~cm} / \mathrm{s}$, only lesser speed is considered.

In order to show the validity of the proposed system, a simple pushing operation without any compliance behavior is conducted. The system response is shown in Fig. 10. It is clearly seen that the position response tightly follow the position command. The collision or the touching stage is represented by sudden change of the estimated force when the robot has moved about $45 \mathrm{~cm}$ from initial location. Please note in Fig. 10, the estimated force is not used as feedback for compliance controller. In addition, the chopstick is broken exactly when the robot body hit it on collision.

Fig. 10 shows that the estimated force rises extremely when the robot start moving and still appears even no contact to the object. Ideally, the external force should be zero until touching the object. One may ask the reliability and the accuracy of the estimated force. Surely, there are some constraints that may disrupt this estimation. As discussed in Section 3, the estimated torque is defined based on estimated friction. Mainly, the estimated friction in Fig. 7 is the average values of each constant velocity. Then, the estimated friction for the

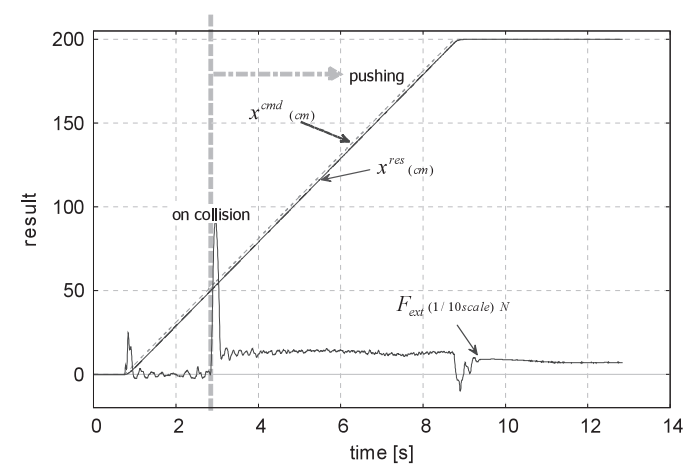

Fig. 10. Pushing without compliance controller 


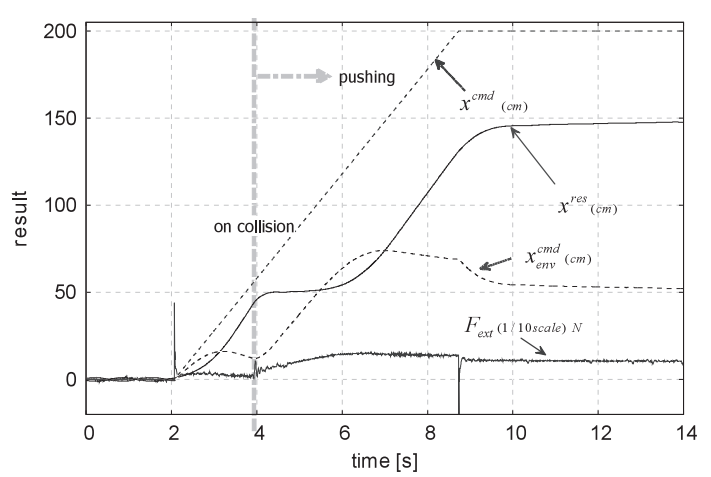

Fig. 11. Pushing without compliance controller

experiment is normalized into (16). In addition, these estimated torques are multiplied with transpose pseudo-inverse Jacobian to convert joint space torques into Cartesian space force. Moreover, the authors find significant gear backslash on both wheels. The friction on floor spot is not similar as well. Hence, the gear backslash, and the estimated friction inaccuracy which is gained by transformation into Cartesian space are defined as the main reasons for any inaccuracy of the estimation result. Furthermore, the momentum of manipulator may give significant disturbance to vehicle part on sudden change in acceleration, as the initial spike appears when robot start moving. Nevertheless, it has good sensation as seen in Fig. 10. A significant spike appears on collision that becomes the main issue in this paper to avoid damage on object or the robot tool-tip on collision during pushing operation.

The following experiment is conducted with compliance controller by using the estimated force $F_{\text {ext }}$. The experimental result is shown in Fig. 11. Since the estimated force grows as the robot move, the compensated position $x_{e n v}^{c m d}$ also gets higher. As a result, the position response is left behind, that is the command reference minus the compensated position. Ideally, the estimated force is zero at approaching stage so that the position response tightly follows the command reference. Inaccuracy of the estimation and slow transient response designed with 1.s settling time are considered as the main reason for this abnormality. Later, the estimated force remains steady after the pushing stage over. This value denotes the static friction of the object about $150 \mathrm{~N}$ which is compensated by compliance controller with $x_{e n v}^{c m d}$ compensation. Unfortunately, the chopstick is broken even with compliance controller. This is because the compensation effectively emerges during touching and pushing. Since the robot moves with $30 \mathrm{~cm} / \mathrm{s}$ before collision, the robot momentum is too big to be hold by the chopstick.

Later in Fig. 12 shows the experimental result with dual compliance controllers. Having non-physical sensation based on visual force, softer touching operation is realized. Here, the chopstick remains all right.

A closer look at Fig. 11 and Fig. 12 shows that the final position response is left behind. Indeed, it is necessary to modify the control structure of Fig. 9, which is adopted from Katsura $^{(8)}$. Obviously, the goal of pushing operation is to make the final position response tightly follow the command reference. On the other hand, the goal of soft touching for softpushing operation is to reduce robot momentum to certain

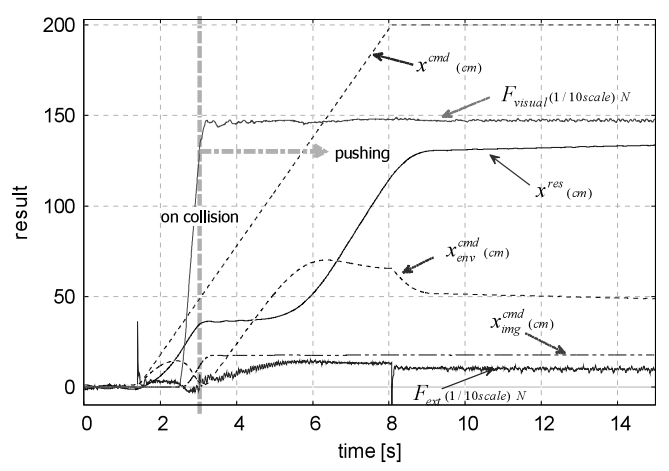

Fig. 12. Pushing compliance with environment and visual force

level when the robot get closer to the object. This is realized by reducing robot speed based on non-contact sensation at approaching stage. Unfortunately, the last result at Fig. 12 shows that the goal of soft touching is realized but not the goal of pushing operation. Refer to Fig. 9, the compensation of compliance controller is actually done at position level (29).

$$
\begin{aligned}
& \ddot{x}^{r e f}=\left(\ddot{x}^{c m d}-\ddot{x}_{i m g}-\ddot{x}_{e n v}\right) \\
& +K_{V}\left(\dot{x}^{c m d}-\dot{x}^{r e s}-\dot{x}_{i m g}-\dot{x}_{e n v}\right) \\
& +K_{P}\left(x^{c m d}-x^{r e s}-x_{i m g}-x_{e n v}\right) \\
& \left(s^{2}+K_{V} s+K_{P}\right) x^{r e s}=\left(s^{2}+K_{V} s+K_{P}\right) x^{c m d} \\
& -\left(s^{2}+K_{V} s+K_{P}\right) x_{i m g} \\
& -\left(s^{2}+K_{V} s+K_{P}\right) x_{e n v} \cdots \\
& x^{r e s}=x^{c m d}-x_{i m g}-x_{e n v}
\end{aligned}
$$

Mainly, the compensation should start at approaching stage just before collision, and then release once the pushing stage over. Thus, the compensation is clearly required when the robot is in a motion. In other words, the controller should have velocity-based compensation. Generally, the compliance controller is not restricted to compensate only displacement, but also velocity as well. A preferable approach is to exclude position component in trajectory compensation.

$$
\begin{aligned}
x^{r e s} & =x^{c m d}-\frac{s^{2}+K_{V} s}{s^{2}+K_{V} s+K_{P}} x_{i m g}-\frac{s^{2}+K_{V} s}{s^{2}+K_{V} s+K_{P}} x_{e n v} \\
& =x^{c m d}-x_{i m g}-x_{e n v}+\frac{K_{P}}{s^{2}+K_{V} s+K_{P}}\left(x_{i m g}+x_{e n v}\right)
\end{aligned}
$$

In which,

$$
\begin{aligned}
\lim _{t \rightarrow \infty} x^{r e s} & =\lim _{s \rightarrow 0} x^{r e s}=x^{c m d}-x_{i m g}-x_{e n v}+1 \cdot\left(x_{i m g}+x_{e n v}\right) \\
& =x^{c m d} \ldots \ldots \ldots \ldots \ldots \ldots \ldots \ldots \ldots \ldots \ldots \ldots \ldots \ldots \ldots \ldots
\end{aligned}
$$

The other approach is to include velocity term of the robot for compliance controllers.

$$
\begin{aligned}
x^{\text {res }} & =x^{\text {cmd }}-\left(x_{i m g}+x_{\text {env }}\right) \dot{x}^{\text {res }} \\
& =x^{\text {cmd }}-\left(\frac{K_{\text {img }}}{Z_{\text {img }}} F_{V}+\frac{K_{f}}{Z_{\text {env }}} F_{e x t}\right) \dot{x}^{r e s} \ldots \ldots \ldots \ldots
\end{aligned}
$$




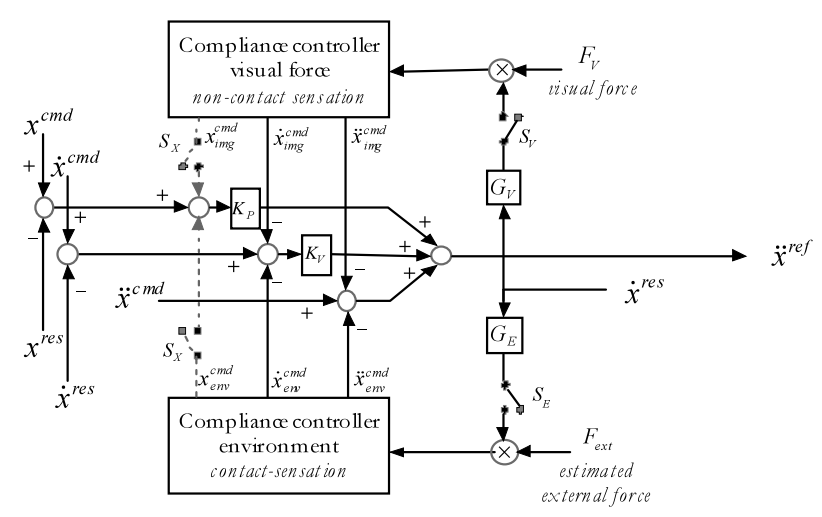

Fig. 13. Adjustment on controller

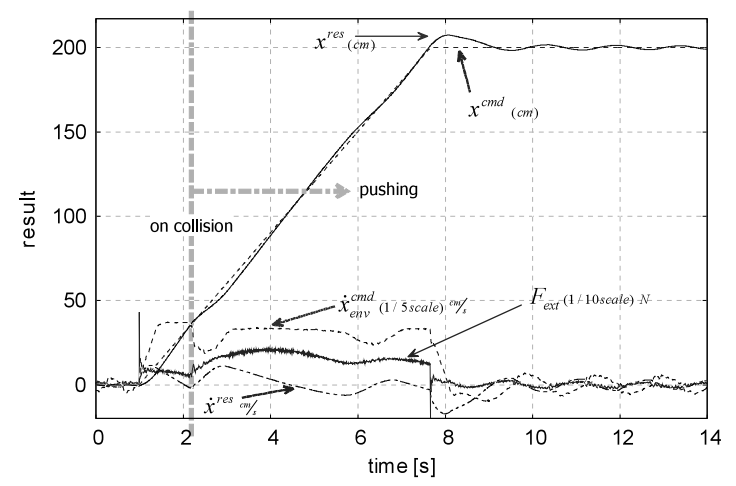

Fig. 14. Env. Compliance with $S_{x}$ OFF

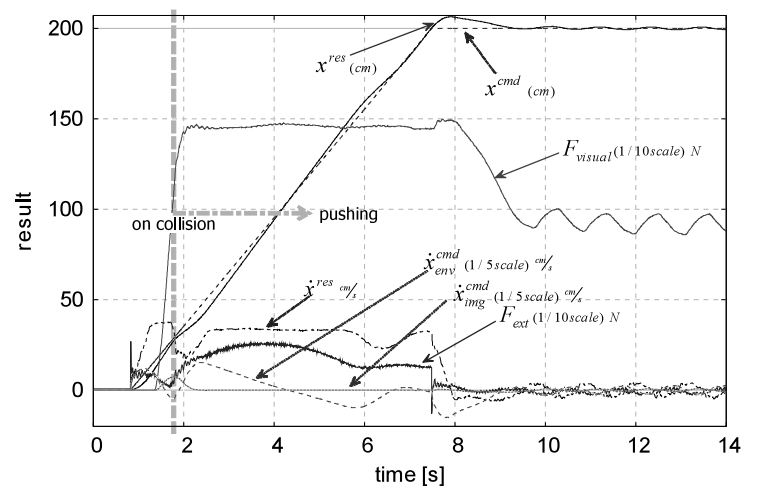

Fig. 15. Dual Compliance with $S_{x}$ OFF

In which, the compensation is active while the robot in a motion.

These lead the authors to adjust the controller as shown in Fig. 13. The position component of both compliance controllers can be excluded by turning off switch $S_{X}$. And the insertion of robot's velocity is performed by turning on switch $S_{E}$ and $S_{V}$.

At first, the exclusion of position component is performed by turning off switch $S_{X}, S_{E}$ and $S_{V}$. The experimental results are shown in Fig. 14 and Fig. 15 for only environmentcompliance controller and for dual compliance controller respectively. The dual compliance controllers' result in Fig. 15 has no significant difference compared to Fig. 14. As seen in Fig. 15, the velocity compensation of non-contact sensation $\dot{x}_{i m g}^{\text {cmd }}$ before collision has insignificant amount compared to robot speed $\dot{x}^{\text {res }}$. Moreover, it may lead to underdamp system as significant ripple appears in the response even after

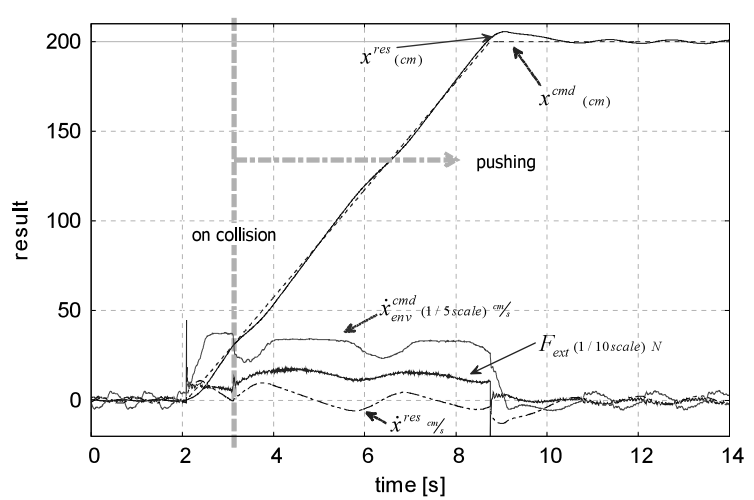

Fig. 16. Env. Compliance with $S_{x}$ OFF with $G_{E}=10$

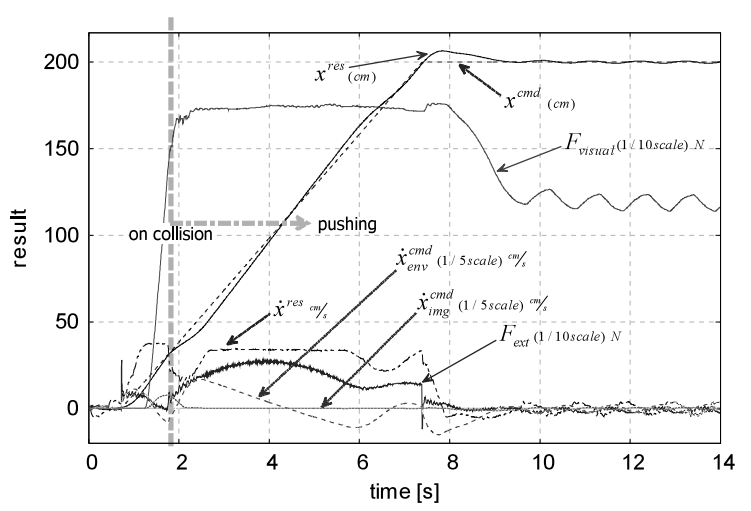

Fig. 17. Dual Compliance with $S_{x}$ OFF with $G_{V}=4$ and $G_{E}=10$

pushing operation has over. By considering (32), further experiments are conducted as shown in Fig. 16 and Fig. 17. The results actually show no difference to results in Fig. 14 and Fig. 15 respectively. Indeed, the idea to exclude position component of compliance controller for trajectory compensation obviously realize the goal of pushing operation but lead to instability. Moreover, soft pushing operation is not effectively realized in this control scheme.

At last, experiments with second approach by applying robot's velocity into compliance controller are conducted. Practically, contact and non-contact compensation can be done independently. Here, equation (32) can be rewrite as,

$$
\begin{aligned}
x^{r e s} & =x^{\text {cmd }}-\left(G_{V} x_{i m g}+G_{E} x_{\text {env }}\right) \dot{x}^{\text {res }} \\
& =x^{\text {cmd }}-\left(G_{V} \frac{K_{\text {img }}}{Z_{\text {img }}} F_{V}+G_{E} \frac{K_{f}}{Z_{\text {env }}} F_{\text {ext }}\right) \dot{x}^{\text {res }} \ldots
\end{aligned}
$$

$G_{V}$ and $G_{E}$ stand for how much the robot velocity play role on compliance controller based on non-contact and contact sensation respectively. The higher these gains, the more compensation emerged on moving robot. Practically, there is no specific rule how to obtain these gains. It depends on how much we need compensation for corresponding sensation while the robot moves. Nevertheless, equation (33) can be rewrite into,

$$
\begin{aligned}
\frac{x^{r e s}}{x^{c m d}} & =\frac{1}{1+G_{V} \frac{K_{i m g}}{Z_{\text {img }}} F_{V} s+G_{E} \frac{K_{f}}{Z_{e n v}} F_{e x t} s} \cdots \cdots \cdots \\
& =\frac{Z_{i m g} Z_{e n v}}{Z_{i m g} Z_{e n v}+\left(G_{V} K_{i m g} F_{V}+G_{E} K_{f} F_{e x t}\right) s}
\end{aligned}
$$




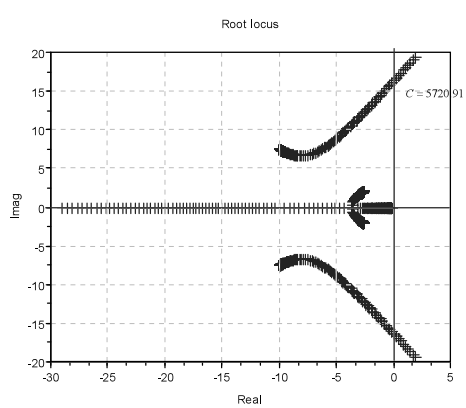

Fig. 18. Root Locus response of eq (35)

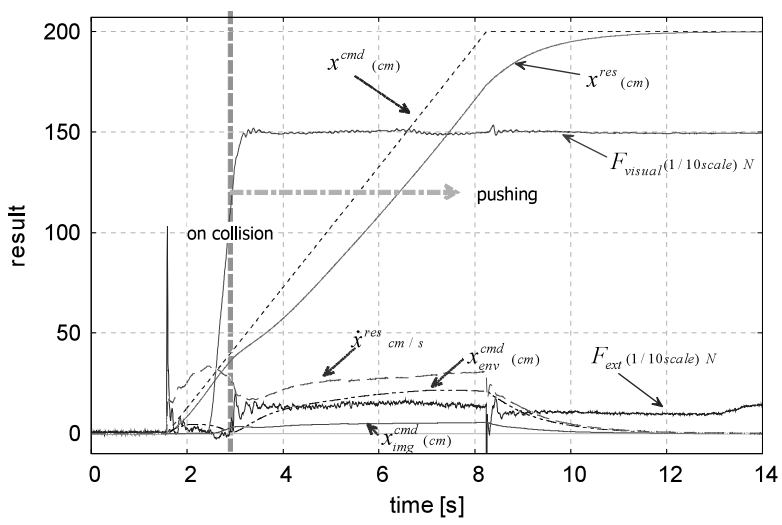

Fig. 19. Dual Compliance with $S_{x}$ ON with $G_{V}=1.0$ and $G_{E}=1.0$

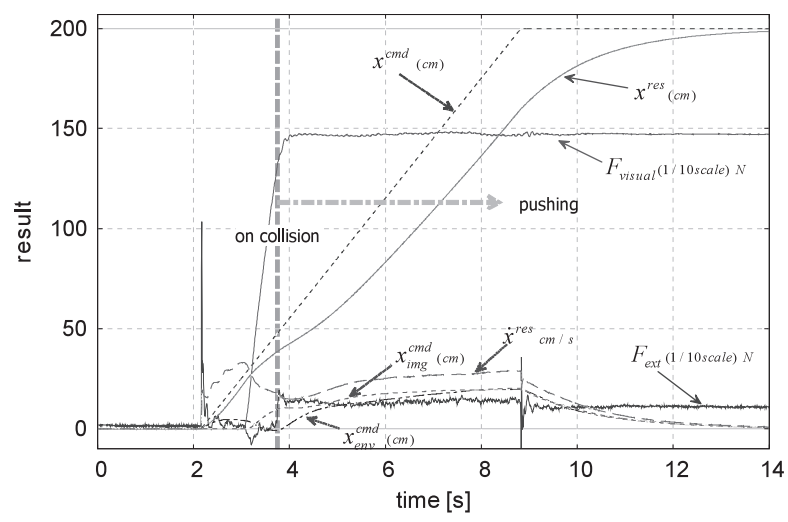

Fig. 20. Dual Compliance with $S_{x}$ ON dwith $G_{V}=4$ and $G_{E}=1$

One may wonder about the system stability since system velocity is used for the compliance controller. Here, the polynomial form transfer function,

$$
\frac{x^{r e s}}{x^{c m d}}=\frac{a_{4} s^{4}+a_{3} s^{3}+a_{2} s^{2}+a_{1} s+a_{0}}{a_{4} s^{4}+a_{3} s^{3}+a_{2} s^{2}+\left(a_{1}+C\right) s+a_{0}} \ldots
$$

where.

$$
C=G_{V} K_{i m g} F_{V}+G_{E} K_{f} F_{\text {ext }}
$$

In which, based on system parameters as listed in Table 1, the root locus system can be observed as shown in Fig. 18. In addition, by using Routh-Hurwitz Stability Criterion, the stability margin is obtained as 5720.91 . Here, by knowing the possible maximum force for the estimated force and visual force, the gain $G_{V}$ and $G_{E}$ can be defined within stability margin. Inspite of that, the controller's characteristic remains the same as these gains practically change $K_{i m g}$ and $K_{f}$ respectively.

The experimental result with both $G_{V}$ and $G_{E}$ set within 1.0 is shown in Fig. 19. Although the response is slower than before, the system is more stable. Moreover, the impact of inaccuracy force estimation due to backlash has gone. Another experiment with $G_{V}$ four times higher than $G_{E}$ is shown in Fig. 20. Here, the non-contact compensation $x_{i m g}^{c m d}$ is much higher on collision, which provides better soft touching. Softer pushing operation practically can be achieved by gaining $G_{V}$. However, the higher this gain, the slower the robot follows the command reference.

\section{Conclusion}

A novel structure for soft pushing operation with dual compliance controllers is proposed in this paper. Two sensations are used in this paper; contact (reaction torque observer) and non-contact (visual sensation) extends the robot ability to sense the object and the environment as well. Both compliance controllers based on those sensors, moderate cooperativeness on object and environmental sensation. In addition, an additional adjustment on controller structure is conducted, so the robot will push the object up to desired location. Having this structure, the damage due to hit's momentum on robot/object surface can be avoided. In addition, the robot will threat the object or the environment moderately. The experimental results confirm the validity of the proposed method.

Obviously, the required effort in this paper may be too tight for simple pushing operation. However, this framework may realize further applications on more complex pushing operation such as pushing operation on space robot where all stuffs should be handled carefully, pushing hazard material, and pushing operation on tele-operation.

Mainly, delay has been a tremendous issue for teleoperation, which often deals with sensitive object. By applying the proposed controller on global bilateral controller in remote site, it may improve tele-operation performance while handling the object/environment moderately. In which, bilateral tele-operation with remote-pushing operation is considered for further work.

\section{Acknowledgment}

This work is supported in part by Hitachi Foundation and a Grant in Aid for the 21st century Center of Excellence for Optical and Electronic Device Technology for Access Network from the Ministry of Education, Culture, Sports, Science, and Technology in Japan.

(Manuscript received July 5, 2006,

revised Nov. 24, 2006)

\section{References}

( 1 ) S. Katsura, J. Suzuki, and K. Ohnishi: "Pushing operation by flexible manipulator taking environmental information into account", Proc. IEEE-AMC (Advanced Motion Control), pp.141-146 (2004)

( 2 ) T. Murakami, R. Nakamura, F. Yu, and K. Ohnishi: "Force Sensorless Compliance Control based on Reaction Force Estimation Observer in MultiDegree-of-Freedom Robot", Journal of RSJ, Vol.11, No.5, pp.756-768 (1993)

( 3 ) A. Verma, J. Boyoon, and G.S. Sukhatme: "Robot box-pushing with environment-embedded sensors", Proc. IEEE Symposium on Computational 
Intelligent in Robotics and Automation, pp.212-217 (2001)

( 4 ) Z. Hongbin, H. Suzuki, T. Nagata, and H. Nagahama: "Coordination of visual and tactile sensors for pushing operation using multiple autonomous arms", Proc. IEEE-Int. Conf. on Multisensor Fusion and Integration for Intelligent Systems, pp.581-588 (1996)

( 5 ) Y. Yu, T. Arima, and S. Tsujio: "Estimation of Object Inertia Parameterson Robot Pushing Operation", Proc. IEEE-ICRA (Int. Conf. on Robotics and Automation), pp.1657-1662 (2005)

( 6 ) T. Kawasa and K. Ohnishi: "Control of pushing operation by a redundant manipulator", Proc. IEEE-AMC (Advanced Motion Control), pp.165-169 (2004)

( 7 ) Y. Ise and T. Murakami: "An Approach to A haptics System Control by Mobile Manipulator", Proc. IEEE-AMC (Advanced Motion Control), pp.239242 (2004)

( 8 ) S. Katsura and K. Ohnishi: "Human Cooperative Wheelchair for Haptic Interaction based on Dual Compliance Control", IEEE Trans. on Industrial Electronics, Vol.51, No.1, pp.221-228 (2004)

(9) A. Muis and K. Ohnishi: "High-speed Visual Tracking Based on Artificial Marker and Hexagon Pattern", Proc. of IEEE-ISIE, Vol.4, pp.1569-1574 (2005)

Abdul Muis (Student Member) was born in Indonesia on 1 September

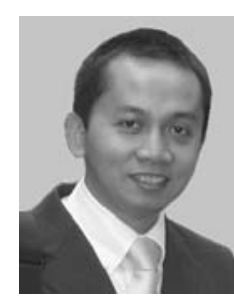
1975. He received B.E. degree in Electrical Engineering from University of Indonesia, Indonesia, in 1998 and received M.E. degree in Integrated Design Engineering from Keio University, Japan, in 2004 and currently Ph.D student in the same University. Since 1999, he has been with Faculty of Engineering, University of Indonesia, Indonesia. His research interests include robotics, image processing, computer engineering, and microprocessors.
Kouhei Ohnishi (Senior Member) was born in Japan on 1952. He re-

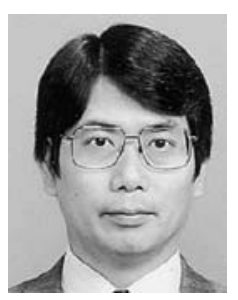
ceived the B.E., M.E., and Ph.D. degrees in electrical engineering from The University of Tokyo, Tokyo, Japan, in 1975, 1977, and 1980, respectively. Since 1980, he has been with Keio University, Yokohama, Japan. His research interests include power electronics, mechatronics, motion control, and robotics. He received the Best Paper Awards from the Institute of Electrical Engineers of Japan and the Japan Society for Precision Engineering, and Outstanding PaperAwards at IECON'85, IECON'92, and IECON'93 from the IEEE Industrial Electronics Society. 